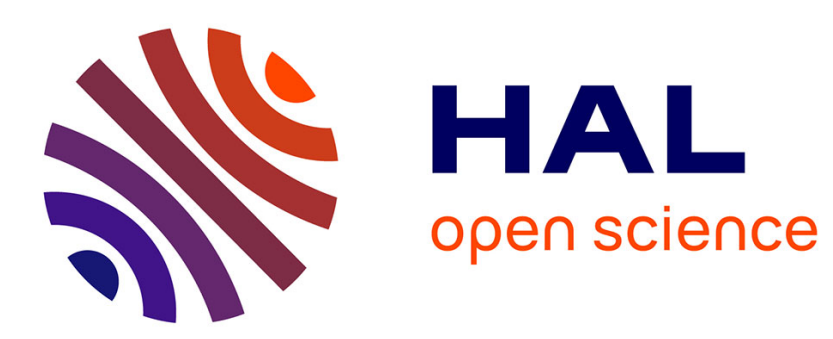

\title{
Phospholipid transverse asymmetry in trout spermatozoa plasma membrane
}

Karin Müller, Catherine Labbé, Alain Zachowski

\section{To cite this version:}

Karin Müller, Catherine Labbé, Alain Zachowski. Phospholipid transverse asymmetry in trout spermatozoa plasma membrane. BBA - Biochimica et Biophysica Acta, 1994, 1192 (1), pp.21-26. 10.1016/0005-2736(94)90138-4 . hal-02703995

\section{HAL Id: hal-02703995 \\ https://hal.inrae.fr/hal-02703995}

Submitted on 1 Jun 2020

HAL is a multi-disciplinary open access archive for the deposit and dissemination of scientific research documents, whether they are published or not. The documents may come from teaching and research institutions in France or abroad, or from public or private research centers.
L'archive ouverte pluridisciplinaire HAL, est destinée au dépôt et à la diffusion de documents scientifiques de niveau recherche, publiés ou non, émanant des établissements d'enseignement et de recherche français ou étrangers, des laboratoires publics ou privés. 


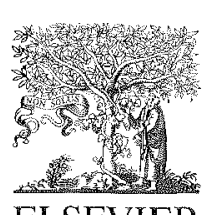

ELSEVIER

\title{
Phospholipid transverse asymmetry in trout spermatozoa plasma membrane
}

\author{
Karin Müller ${ }^{\mathrm{a}, 1}$, Catherine Labbé ${ }^{\mathrm{b}}$, Alain Zachowski ${ }^{\mathrm{a}, *}$ \\ "Institut de Biologie Physico-Chimique; 13, rue Pierre et Marie Curie, 75005 Paris, France \\ ${ }^{b}$ Laboratoire de Physiologie des Poissons, INRA, Campus de Beaulieu, 35042 Rennes, France
}

(Received 5 October 1993; revised manuscript received 26 January 1994)

\begin{abstract}
The phospholipid transmembrane distribution and movement in the plasma membrane of rainbow trout spermatozoa was determined with spin-labelled phospholipid analogues. After initial incorporation in the outer membrane leaflet, only the aminophospholipids, phosphatidylserine (PS) and phosphatidylethanolamine (PE) redistributed towards the inner leaflet. At equilibrium, more than $90 \%$ PS and approximately $80-85 \%$ PE were located in the cytoplasmic leaflet. The inward motion was significantly faster for $\mathrm{PS}\left(t_{1 / 2} \approx 5 \mathrm{~min}\right)$ than for $\mathrm{PE}\left(t_{1 / 2} \approx 60 \mathrm{~min}\right)$. Both the velocity and the extent of this redistribution were dependent on the cellular ATP level, arguing for the involvement of the aminophospholipid translocase. Comparison of the electron paramagnetic resonance spectra recorded from analogues located in either one of the leaflet showed an important difference of microviscosity between the cytoplasmic and the extracellular bilayer leaflets. Assuming that the analogue distribution reflects the asymmetry of the endogenous phospholipids, the higher probe mobility in the inner leaflet could be correlated to a higher degree of unsaturation of the fatty acids present in this hemi-leaflet.
\end{abstract}

Key words: Phospholipid; Asymmetry; Spermatozoa; Fluidity; Aminophospholipid translocase; (Trout)

\section{Introduction}

All the reported studies on the disposition of phospholipids between the two halves of eukaryotic plasma membranes show, with a few exceptions, that the choline phospholipids sphingomyelin (SM) and phosphatidylcholine (PC) are the main components of the external leaflet while the aminophospholipids, phosphatidylserine (PS) and phosphatidylethanolamine (PE) are principally found in the cytoplasmic leaflet $[1,2]$. This transmembrane asymmetry results from the activity of the aminophospholipid translocase which transports PS and PE molecules from the outer to the inner leaflet at the expense of cytoplasmic ATP hydrolysis [3-5]. Their accumulation at the cytoplasmic sur-

\footnotetext{
* Corresponding author. Fax: +33 1 40518317. E-mail: zachowski@ibpc.fr.

${ }^{1}$ Present address: Institut für Fortpflanzung landwirtschaftlicher Nutztiere Schönow e.V., 16321 Schönow, Germany.
}

face does not require any interaction with extrinsic proteins but is the result of an equilibrium between a fast inward movement and a slow, passive outward diffusion [5-7]. PS is translocated faster to and is present in larger amount in the cytoplasmic monolayer than PE because the translocase exhibits approximately a 10-times higher affinity for this lipid [8]. Moreover, the degree of accumulation of PS and PE on the inner face depends on the cellular ATP concentration [5,9].

One exception to this general scheme are myoblasts which are going to fuse into a myotube [10]. In this case, the phospholipid distribution is almost symmetrical, and the outer leaflet contains an appreciable amount of PS and PE. This disposition was supposed to be adapted to cell fusion as the aminophospholipids are recognized to be fusogenic. One can thus wonder which is the transverse phospholipid composition of a spermatozoa plasma membrane as, during the fertilization process, this cell will fuse with the oocyte. Reported data on the topology of ram [11] and guinea pig [12] spermatozoa plasma membrane showed that the aminophospholipids do not exhibit a highly asymmetric 
transverse distribution. On the contrary, goat sperm plasma membrane exhibits a pronounced PE asymmetry [13]. Here, we have studied the transverse movement and distribution of phospholipid analogues in the rainbow trout spermatozoa. Trout spermatozoa are devoid of acrosome and fertilization occurs solely by fusion of oocyte and spermatozoa membranes at the micropylar site. The spin-labelled analogues utilized in this study have proved to be reliable reporters of their endogenous counterparts in many cell membranes, as they mimic both the diffusion rates and the steady-state asymmetry of the long-chain diacyl molecules [14-16]. We describe the existence of an aminophospholipid translocase in the trout spermatozoa membrane, and the asymmetric distribution of the phospholipids across the bilayer. The transport rate and asymmetry of PS strictly depend on the cellular ATP content. On the other hand, the diffusion of the choline lipids between the monolayers is insignificant. This polar headgroup asymmetry is accompanied by a difference in the fatty acyl chain composition of each leaflet which creates a drastic gradient of fluidity through the bilayer.

\section{Materials and methods}

Spermatozoa. Fresh semen was obtained by aspiration from fishes anaesthezied by phenoxy-2-ethanol [17]. The cell suspension was kept under an oxygenated atmosphere at $4^{\circ} \mathrm{C}$ until use.

Phospholipid class determination. Plasma membranes were purified as previously described [18]. Lipid extraction was carried out following Folch et al. [19] with some modifications. To one vol. of membrane suspension were added 4 vol. of $\mathrm{CHCl}_{3} / \mathrm{CH}_{3} \mathrm{OH}(2: 1, \mathrm{v} / \mathrm{v})$ supplemented with $0.02 \%(\mathrm{w} / \mathrm{w})$ butyl hydroxytoluene $(\mathrm{BHT})$. After vigorous agitation, the mixture was centrifuged for $10 \mathrm{~min}$ at $1000 \times \mathrm{g}$. The bottom phase was then dried under a nitrogen stream and dissolved in $\mathrm{CHCl}_{3}+\mathrm{BHT}$. The phospholipid fraction was isolated from the other lipid fractions by chromatography on a Sep-Pak silica cartridge (Waters Chrom. Div., Millipore) according to Juaneda and Rocquelin [20]. Different phospholipid classes were separated by high performance liquid chromatography on a Zorbax silica column $(250 \times 4.6 \mathrm{~mm}, 5 \mu \mathrm{m}$ granulometry; Société Française de Chromatographie) by varying the mobile phase from hexane/isopropanol $(3: 2, \mathrm{v} / \mathrm{v})$ to the same mixture containing $5.5 \%$ vol. of water (Labbé, to be published). Purity of each phospholipid fraction was checked by thin-layer chromatography. After trans methylation, samples were submitted for gaseous phase chromatography on a silica capillary column $(50 \mathrm{~m}$ long, $0.25 \mathrm{~mm}$ internal diameter, CP WAX $52 \mathrm{CB}$ Chrompack). Methylated esters were identified by comparison with standards.
Spin labelling and EPR determination of the transmembrane distribution of spin-labelled lipids. Spermatozoa were washed twice $\left(15 \mathrm{~min}, 500 \times \mathrm{g}, 4^{\circ} \mathrm{C}\right)$ in a phosphate-buffered saline solution $(90 \mathrm{mM} \mathrm{NaCl}, 29$ $\mathrm{mM} \mathrm{KCl}, 1.1 \mathrm{mM} \mathrm{MgSO}_{4}, 10 \mathrm{mM} \mathrm{Na-succinate,} 10$ $\mathrm{mM} \mathrm{Na}_{2} \mathrm{HPO}_{4}, 20 \mathrm{mM}$ Tris- $\mathrm{HCl}$ (pH 8)). Immediately before incubation, the cell suspension was supple-mented with $5 \mathrm{mM}$ diisopropylfluorophosphate to minimize spin-labelled phospholipid hydrolysis [15]. 1*Palmitoyl-2-(4-doxylpentanoyl)phosphatidylcholine ( $\mathrm{PC}^{*}$ ), -phosphatidylserine (PS*), -phosphatidylethanolamine (PE*) and $N$-(4-doxylpentanoyl)-trans-sphingenyl-1phosphocholine $\left(\mathrm{SM}^{*}\right)$ were synthesised as described [21]. An aliquot of the desired analogue in chloroform solution, corresponding to $1 \%$ of the endogenous membrane phospholipids in the final incubation, was dried under vacuum and resuspended by vigorous vortexing with buffer. Cell sample and label suspension were prewarmed at $20^{\circ} \mathrm{C}$ and the translocation assay was initiated by mixing 1 vol. of phospholipid suspension to 2 vol. of cell suspension at $10^{10}$ cells $/ \mathrm{ml}$. Determination of the lipid transmembrane distribution was performed by the back exchange technique [21]. Briefly $120 \mu \mathrm{l}$ aliquots were taken from the labelled cell suspension at given times, mixed with $30 \mu 1$ of $4 \%$ fatty acid-free BSA and incubated on ice for $1 \mathrm{~min}$. After centrifugation (30 s, 7600 $\times g$ in an Eppendorf tube), the supernatant was taken and analysed by electron spin resonance (EPR) spectroscopy after addition of $10 \mathrm{mM}$ potassium hexaferricyanide to reoxidize all of the label. Comparison of the signal associated to the pellets of aliquots treated at time zero with and without BSA demonstrated that more than $97 \%$ of the analogue were recovered in the BSA-containing supernatant. Data were fitted with exponential equations using the KaleidaGraph data analysis and graphics application (Abelbeck Software).

Cyanide treatment. A kinetic study of $\mathrm{PC}^{*}$ and $\mathrm{PS}^{*}$ movements in the membrane was carried out as above. After 45 min of incubation, an aliquot of each sample was taken out and supplied with $15 \mathrm{mM} \mathrm{KCN} \mathrm{in} \mathrm{order}$ to poison the mitochondrion and lower the cellular ATP content. The relocation of both probes was followed both on the treated and the untreated samples. In parallel, an unlabelled cell suspension was treated by $\mathrm{KCN}$ for $30 \mathrm{~min}$ prior to addition of phospholipid analogues and the determination of the transmembrane movement in such ATP-depleted cells.

Recording of the membrane spectra. The spin-labelled lipids were introduced into the spermatozoa membranes as for the kinetics assay. After a 30 min incubation at $20^{\circ} \mathrm{C}$, cells were pelleted by centrifugation and mixed with potassium hexaferricyanide (final concentration $10 \mathrm{mM}$ ). All EPR measurements were per. formed with a Varian E-109 spectrometer equipped with a temperature control device. 
Miscellaneous. Cell lysis was estimated by assaying the lactate dehydrogenase (LDH) activity present in the supernatant of a centrifuged cell sample. In all the reported experiments, lysis was less than $3 \%$. Statistical comparisons were done using a $t$-test (paired observation, $\alpha=0.05$ ). Cell ATP content was determined by the luciferin-luciferase assay (LKB 1243-200 ATP monitoring reagent kit).

\section{Results}

\subsection{Transmembrane movement of spin-labelled phospho- lipids}

After incorporation in the outer leaflet, the behaviour of the spin-labelled analogues differed clearly according to the nature of the polar headgroup (Fig. 1). The cholinephospholipids $\mathrm{PC}^{*}$ and $\mathrm{SM}^{*}$ remained in the outer monolayer, no significant redistribution into the inner one being measurable over the incubation time. On the other hand, the aminophospholipids quickly left the exofacial layer and accumulated in the cytoplasmic one. This redistribution was rapid for PS* and required several hours for PE*. When studied over at least six independent experiments, less than $5 \%$ of either $\mathrm{PC}^{*}$ or $\mathrm{SM}^{*}$ was moved through the bilayer, while $91.2 \pm 2.9 \%$ of PS* and $84.3 \pm 1.8 \%$ of $\mathrm{PE}^{*}$ were at equilibrium in the inner monolayer. In order to assess the involvement of the aminophospholipid translocase activity in the differential behaviour, relocalization kinetics were repeated in cells with different amounts of cytoplasmic ATP. The source of ATP syn-

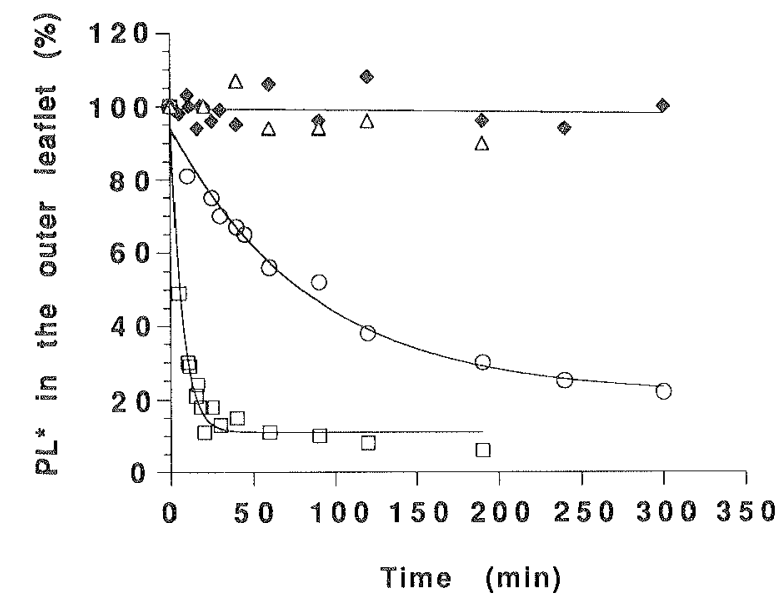

Fig. 1. Transmembrane relocation of spin-labelled phospholipid analogues initially inserted in the outer leaflet. At given times, aliquots of cell suspension were sampled out and the fraction of analogue present in the outer leaflet was assayed by back-exchange on bovine serum albumin (see Materials and methods). Data presented here represent a typical experiment. PC* $(\$), \mathrm{PS}^{*}(\square), \mathrm{PE}^{*}(\mathrm{O})$ and $\mathrm{SM}^{*}(\triangle)$. Curves were fitted by Kaleidagraph (Abelbeck Software) programme.

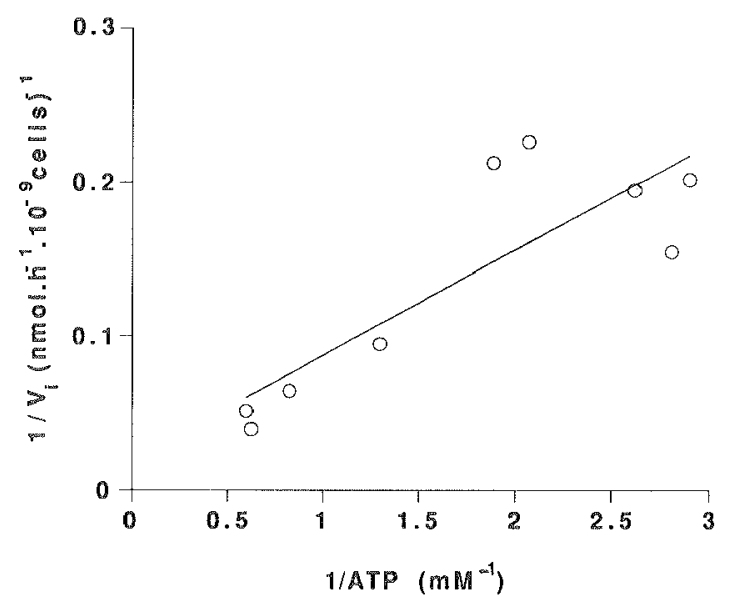

Fig. 2. Lineweaver-Burk representation of the initial rate of $\mathrm{PS}^{*}$ reorientation to the membrane inner leaflet as a function of the cytoplasmic ATP content. Rates were determined from curves similar to those shown in Fig. 1 obtained with cells of varied cytoplasmic ATP concentrations after storage in oxygen-free conditions for varying length of time.

thesis being the unique spermatozoa mitochondrion, ATP concentration could be manipulated by incubating the cells for different length of time in oxygen-free conditions. Lowering the nucleotide content induced a slower redistribution of PS* towards the cytoplasmic leaflet (Fig. 2). The apparent $K_{\mathrm{m}}$ for ATP appeared to be $3.58 \pm 0.78 \mathrm{mM}$. Similarly, the steady-state distribution of PS* was very sensitive to the cytoplasmic ATP, as when ATP dropped below $0.4 \mathrm{mM}$ the asymmetric PS* distribution was less pronounced (Fig. 3). To verify that a drop in cytoplasmic ATP will affect the distribution of analogues previously equilibrated in the bilayer, cells were treated by $15 \mathrm{mM} \mathrm{KCN}$ which reduced the cytoplasmic ATP level to $20.1 \pm 2.9 \%$ of its original value. This induced a redistribution of some PS* in

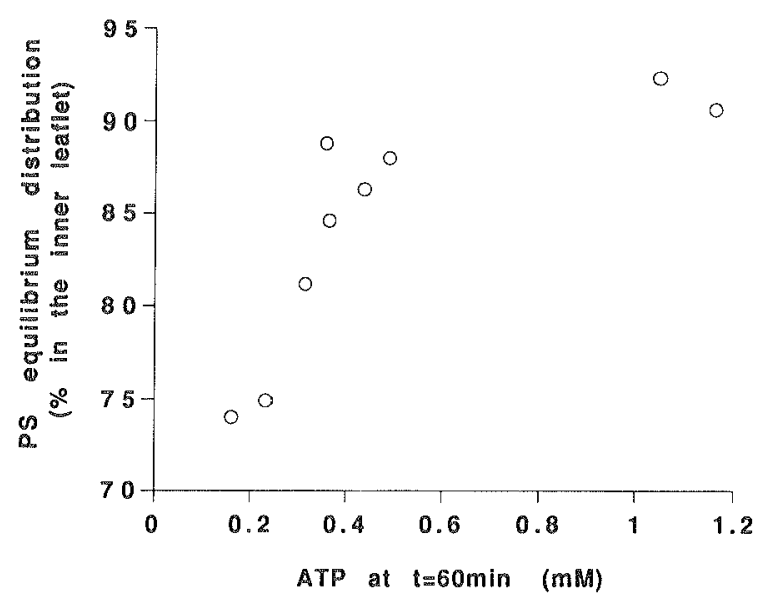

Fig. 3. Steady-state distribution of PS* analogue in spermatozoa plasma membrane according to cytoplasmic ATP concentrations. The nucleotide content is given after one hour of incubation, i.e., once the equilibrium was reached under any condition. 


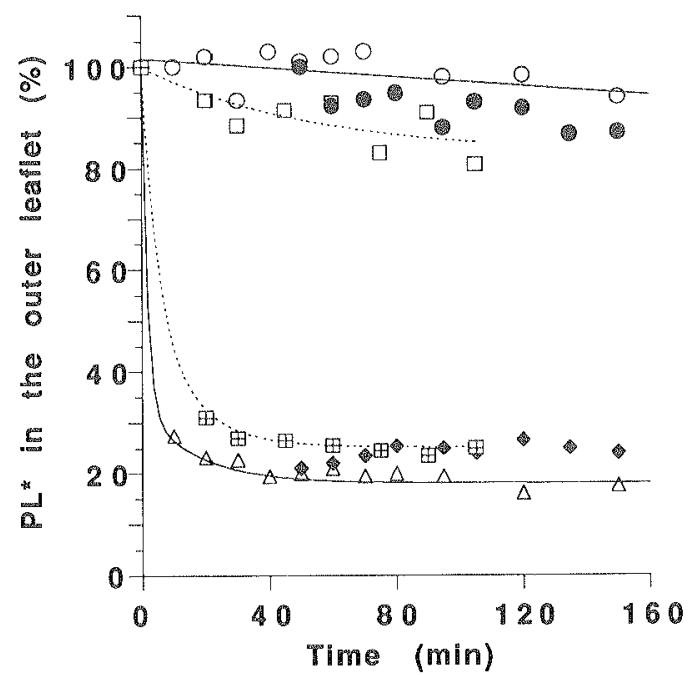

Fig. 4. PC* $(O)$ and $\mathrm{PS}^{*}(\Delta)$ analogues were added to sperm cells and allowed to equilibrate within the bilayer. After a 45 min incubation, part of the cells were treated by $\mathrm{KCN}$ and the reorientation of $\mathrm{PC}^{*}$ (*) and $\mathrm{PS}^{*}($ ) was followed with time. In parallel, a cell suspension has been treated by KCN prior to monitor the transmem. brane movement of PC* $(\square)$ and PS* (⿴).

favour of the outer membrane leaflet and a movement of $\mathrm{PC}^{*}$ toward the inner monolayer (Fig. 4). The new PS* distribution was identical to the one obtained with cells which were first poisoned prior to studying the analogue transbilayer movement.

\subsection{Acyl chain composition of membrane halves}

The phospholipid composition of the plasma membrane was determined with isolated membranes. When assayed over seven different preparations, the plasma membrane contained $3.4 \pm 0.8 \%$ phosphatidylinositol (PI), $1.5 \pm 1.1 \% \mathrm{SM}, 31.8 \pm 1.6 \% \mathrm{PE}, 9.8 \pm 0.7 \% \mathrm{PS}$,
$49.5 \pm 3.1 \%$ PC and minor compounds (lysoderivatives for instance). Considering that the asymmetry determined with the spin-labelled analogues applies to the endogenous molecules, the composition of the outer leaflet, expressed in mole fraction of the total phospholipids, would be $0.48 \mathrm{PC}, 0.01 \mathrm{SM}, 0.05 \mathrm{PE}$ and 0.01 PS. The inner leaflet would contain the remaining population (0.02 PC, 0.10 PS, 0.28 PE). From these numbers, one can hypothezise that PI should be located in the cytoplasmic leaflet, in order to obtain almost equally populated membrane leaflets.

For each phospholipid class, the fatty acyl chain content was determined; the distribution of saturated, mono-unsaturated and poly-unsaturated chains for the lipids under study is shown in Table 1. Knowing the phospholipid composition of the two monolayers allows for the deduction of their fatty acyl chain composition (Table 2). The outer leaflet contains more saturated chains and less poly-unsaturated ones than its inner counterpart.

\subsection{Membrane microviscosity}

We compared the EPR spectra recorded with spin-

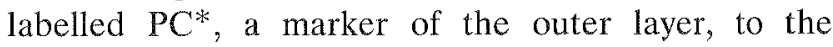
spectra originating from spin-labelled PS*, which was at more than $90 \%$ in the inner leaflet (Fig. 5). Spectra were normalized to a same probe concentration; under this condition, a spin-labelled molecule with a greater mobility gives rise to a spectrum with higher and narrower lines. Reported spectra show that, at a given temperature, the $\mathrm{PC}^{*}$ probe possessed a much lower mobility than the PS* one, showing a more rigid outer plasma membrane leaflet. Comparison of spectra recorded at various temperature indicates that, to ex-

Table 1

Fatty acid class distribution among the main phospholipids of the trout spermatozoa plasma membrane

\begin{tabular}{llllll}
\hline $\begin{array}{l}\text { Phospholipid } \\
\text { class }\end{array}$ & $\begin{array}{l}\text { SFA } \\
(\%)\end{array}$ & $\begin{array}{l}\text { MUFA } \\
(\%)\end{array}$ & $\begin{array}{l}\text { PUFA } \\
(\%)\end{array}$ & UFA/SFA & UI \\
\hline PC & $44.6 \pm 0.5$ & $25.4 \pm 0.1$ & $30.0 \pm 0.5$ & $1.24 \pm 0.02$ & $171.2 \pm 1.6$ \\
PE & $26.5 \pm 0.8$ & $22.4 \pm 0.6$ & $51.1 \pm 0.2$ & $2.77 \pm 0.11$ & $265.4 \pm 2.5$ \\
PS & $19.4 \pm 1.5$ & $36.7 \pm 3.0$ & $43.9 \pm 1.5$ & $4.15 \pm 0.40$ & $255.6 \pm 5.6$ \\
PI & $41.5 \pm 0.4$ & $21.6 \pm 3.0$ & $36.9 \pm 3.5$ & $1.41 \pm 0.02$ & $190.3 \pm 14.3$
\end{tabular}

SFA: saturated fatty acids; MUFA: mono-unsaturated fatty acids; PUFA: poly-unsaturated fatty acids; UFA: unsaturated fatty acids. The unsaturation index (UI) was calculated as the sum of the proportion of each unsaturated fatty acid multiplied by its double bond number. Reported values are the average of 4 different determinations, each with a mixture of spermatozoa taken from 5 trouts.

Table 2

Fatty acid chain composition of the plasma membrane hemi-leaflets

\begin{tabular}{|c|c|c|c|c|c|}
\hline & SFA & MUFA & PUFA & UFA/SFA & UI \\
\hline External leaflet & 42.5 & 25.3 & 32.2 & 1.35 & 181.3 \\
\hline Internal leaflet & 26.7 & 25.8 & 47.5 & 2.75 & 253.8 \\
\hline
\end{tabular}

Values were calculated from the distribution of each phospholipid class within the membrane as determined with spin-labelled analogues (see Results section) and from the fatty acid content of each species (see Table 1). Data are expressed in $\%$ of class in the hemi-leaflet. For abbreviations, see Table 1. 


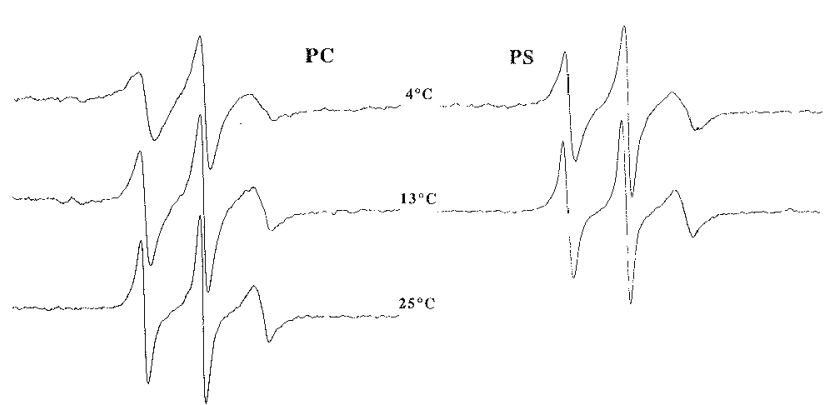

Fig. 5. EPR spectra recorded with spin-labelled phospholipid analogues present in the outer $\left(\mathrm{PC}^{*}\right)$ or the inner (PS*) leaflet of spermatozoa plasma membrane at different temperature. Spectra were recorded on a Varian E109 spectrometer. Scan width $100 \mathrm{G}$; amplitude modulation $1 \mathrm{G}$; microwave power $10 \mathrm{~mW}$.

hibit a viscosity similar to the inner leaflet one, the outer leaflet has to be over-heated by approx. $10 \mathrm{C}^{\circ}$ (compare the $\mathrm{PC}^{*}$ spectra recorded at $13^{\circ} \mathrm{C}$ and $25^{\circ} \mathrm{C}$ to the $\mathrm{PS}^{*}$ spectra recorded at $4^{\circ} \mathrm{C}$ and $13^{\circ} \mathrm{C}$, respectively).

\section{Discussion}

Like many plasma membranes, that of trout spermatozoa is asymmetric regarding its phospholipid composition. The choline lipid $\mathrm{PC}^{*}$ (SM being only a very minor class) is found almost totally in the exofacial leaflet. This distribution is similar to the one found in mammalian spermatozoa [11-13]. The two aminophospholipids, $\mathrm{PS}^{*}$ and $\mathrm{PE}^{*}$, are located primarily in the cytoplasmic leaflet. This result is close to the situation encountered in goat sperm cells [13] but is clearly different to the asymmetry described in ram and guinea pig cells $[11,12]$ in which these phospholipids are almost symmetrically distributed in the bilayer. The authors proposed that this latter distribution should correspond to the specific function of the spermatozoa plasma membrane connected with the capacitation and the fusion through the acrosome reaction. However trout spermatozoa are immobile in the sperm duct, as the motility is initiated by dilution of external $\mathrm{K}^{+}$when cells are released in water. The distribution described here corresponds to an unstimulated cell, which is deprived from acrosome, and thus whose capacitation will occur by a different mechanism. In order to assume an equilibrium in mass of these two leaflets, one has to consider that PI is also asymmetrically distributed in favour of the inner layer. This distribution corresponds to the one found in the human erythrocyte membrane $[22,23]$.

The $\mathrm{PS}^{*}$ and $\mathrm{PE}^{*}$ asymmetry is due to the activity of the aminophospholipid translocase present in the membrane. Indeed, the rate of inward relocation of PS* is dependent on the cytoplasmic ATP concentra- tion. The apparent affinity for the nucleotide is somewhat higher than in the human erythrocyte [3], but remains in the same range ( $3 \mathrm{mM}$ compared to $1 \mathrm{mM}$ ). As in red cells [5,9], the steady-state distribution of PS* is also dependent on the cytoplasmic ATP: a low ATP content only supported a low asymmetrical distribution of the phospholipid. In addition, a drop in cytoplasmic ATP is followed by a reduction of the phospholipid asymmetry, as could be seen in cells which have been poisoned. This can be explained by the fact that the transversal equilibrium disposition of the lipids results from a kinetics balance between an inward active movement and an outward passive one [6,7]. Not only did lowering the cytoplasmic ATP slow the rate of

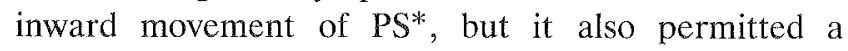
passive inward movement of $\mathrm{PC}^{*}$ which explains the less asymmetrical distribution obtained in these conditions. We were not able to study the movement of all the phospholipids in cells totally depleted of ATP as under these conditions the spin-labelled analogues were hydrolyzed at a high rate, probably due to the fact that they resemble shortened, peroxydized molecules which are to be treated by a de-acylase system, certainly present in these cells [24]. This system would have to be more active as cells lost their ATP. The sensitivity of the spin-probes to cellular phospholipase $\mathrm{A}_{2}$-like enzymes is not peculiar to the spermatozoa [25].

As in the human erythrocyte, the acyl-chain composition depends on the polar headgroup [26]. The choline phospholipids contain more saturated chains than the aminophospholipids, the reverse being true for the polyunsaturated chains. As a consequence, the outer and the inner leaflets differ in their degree of unsaturation, the cytoplasmic one being the leaflet with the more unsaturations. One can thus expect that the spectra recorded with spin-labelled phospholipids embedded in one or the other membrane halves would reflect a different rotational mobility. Indeed, the PS* probe gave rise to a narrower EPR spectrum than the $\mathrm{PC}^{*}$ probe at a given temperature. This is the sign of a higher fluidity of the cytoplasmic leaflet when compared to the exofacial one. Such a result has been described in human erythrocyte membrane [25]. In this plasma membrane [16], as well as in fibroblasts [27], it appeared that the phospholipids could diffuse more easily in the plane of the inner monolayer than in the plane of the outer one. We thus presume that it will be also the case in the spermatozoa plasma membrane.

An external monolayer containing cholinephospholipids [1,2] and relatively rigid [28] is not favourable to cell fusion. It would rather require the outward appearance of PS and PE, which are fusogenic and which would decrease the leaflet rigidity. However, emission of the spermatozoa in water induces a drop in cytoplasmic ATP [29] and an uptake of calcium ions [30]. Such an increase in cytoplasmic calcium should favour a 
bilayer scrambling during which PS and PE would be relocalized in the outer leaflet, as it happens in erythrocytes [31,32] and platelets [33]. Moreover, ATP depletion and high calcium in the cytoplasm will inhibit the aminophospholipid translocase and prevent it from returning the aminophospholipids into the cytoplasmic leaflet $[6,34]$.

\section{Acknowledgements}

We are indebted to Drs. P.F. Devaux, M. Loir and G. Maisse for their stimulating discussions, Ms. P. Hervé for providing us with the spin-labelled phospholipids and Ms. M. Auland for reading the manuscript. Work was supported by grants from the Centre National de la Recherche Scientifique (URA 526), the Institut National de la Recherche Agronomique and the Universite Paris 7. K.M. was a recipient of an E.M.B.O. short-term fellowship.

\section{References}

[1] Devaux, P.F. (1992) Annu. Rev. Biophys. Biomol. Struct. 21, 417-439.

[2] Zachowski, A. (1993) Biochem. J. 294, 1-14.

[3] Seigneuret, M. and Devaux, P.F. (1984) Proc. Natl. Acad. Sci. USA $81,3751-3755$.

[4] Daleke, D.L. and Huestis, W.H. (1985) Biochemistry 24, 54065416.

[5] Beleznay, Z., Zachowski, A., Devaux, P.F., Puente Navazo, M. and Ott, P. (1993) Biochemistry 32, 3146-3152.

[6] Bitbol, M. and Devaux, P.F. (1988) Proc. Natl. Acad. Sci. USA 85, 6783-6787.

[7] Connor, J., Pak, C.H., Zwaal, R.F.A. and Schroit, A.J. (1992) J. Biol. Chem. 267, 19412-19417.

[8] Zachowski, A., Favre, E., Cribier, S., Hervé, P. and Devaux, P.F. (1986) Biochemistry 25, 2585-2590.

[9] Calvez, J.-Y., Zachowski, A., Hermann, A., Morrot, G. and Devaux, P.F. (1988) Biochemistry 27, 5666-5670.

[10] Sessions, A. and Horwitz, A.F. (1983) Biochim. Biophys. Acta 728, 103-111.
[1.1] Hinkovska, V.T., Dimitrov, G.P. and Koumanov, K.S. (1986) Int. J. Biochem. 18, 1115-1121.

[12] Elliot, M. and Higgins, J.A. (1983) Cell Biol. Int. Rep. 7, 1091-1096.

[13] Rana, A.P.S., Misra, S., Majumder, G.C. and Ghosh, A. (1993) Biochim. Biophys. Acta 1210, 1-7.

[14] Tilley, L., Cribier, S., Roelofsen, B., Op den Kamp, J.A.F. and Van Deenen, L.L.M. (1986) FEBS L.ett. 194, 21-27.

[15] Daleke, D.L. and Huestis, W.H. (1989) J. Cell Biol. 108, 13751385.

[16] Morrot, G., Hervé, P., Zachowski, A., Fellmann, P. and Devaux, P.F. (1989) Biochemistry 28, 3456-3462.

[17] Maisse, G., Pinson, A. and Loir, M. (1988) Aquat. Living Res. 1, $45-51$.

[18] Labbé, C. and Loir, M. (1991) Fish Physiol. Biochem. 9, 325-338.

[19] Folch, J., Lees, M. and Sloane-Stanley, G.M. (1957) J. Biol. Chem. 226, 497-509.

[20] Juaneda, P. and Rocquelin, G. (1985) Lipids 20, 40-41.

[21] Fellmann, P., Zachowski, A. and Devaux, P.F. (1994) in Methods in Molecular Biology, Vol. 27, Biomembrane Protocols: II. Architecture and Function (Graham and Higgins, eds.), pp. 161-175, Humana Press.

[22] Bütikofer, P., Lin, Z.W., Chiu, D.T.-Y., Lubin, B. and Kuypers, F.A. (1990) J. Biol. Chem. 265, 16035-16038.

[23] Gascard, P., Tran, D., Sauvage, M, Sulpice, J.-C., Fukami, T., Claret, M. and Giraud, F. (1991) Biochim. Biophys. Acta 1069, $27-36$.

[24] Langlais, J. and Roberts, K.D. (1985) Gamete Res. 12, 183-224.

[25] Seigneuret, M., Zachowski, A., Herrmann, A. and Devaux, P.F. (1984) Biochemistry 23, 4271-4275.

[26] Myher, J.J., Kuskis, A. and Pind, S. (1989) Lipids 24, 396-407.

[27] El-Hage Chahine, J.-M., Cribier, S. and Devaux, P.F. (1993) Proc. Natl. Acad. Sci. USA 90, 447-451.

[28] Herrmann, A., Clague, M.J., Puri, A., Morris, S.J., Blumenthal, R. and Grimaldi, S. (1990) Biochemistry 29, 4054-4058.

[29] Christen, R., Gatti, J.-L. and Billard, R. (1987) Eur. J. Biochem. $166,667-671$.

[30] Cosson, M.-P., Billard, R. and Letellier, L. (1989) Cell Motil. Cytoskel. 14, 424-434.

[31] Williamson, P., Algarin, L., Bateman, J., Choe, H.-R. and Schlegel, R.A. (1985) J. Cell. Physiol. 123, 209-214.

[32] Williamson, P., Kulick, A., Zachowski, A., Schlegel, R.A. and Devaux, P.F. (1992) Biochemistry 31, 6355-6360.

[33] Schroit, A.J. and Zwaal, R.F.A. (1991) Biochim. Biophys. Acta $1071,313-329$.

[34] Comfurius, P., Senden, J.M.G., Tilly, R.H.J., Schroit, A.J., Bevers, E.M. and Zwaal, R.F.A. (1990) Biochim. Biophys. Acta $1026,153-160$. 